\title{
PADDY DISEASES RECOGNITION USING CONVOLUTIONAL NEURAL NETWORK
}

\section{Sony Annem}

Department of Computer Science

SreeNidhi Institute of science and technology, Hyderabad

\begin{abstract}
Agriculture is one of the ancient methods to produce food by cultivating plants. Many countries depend on agriculture sector. Paddy is the largest growing in agriculture sector. Paddy is one of the most essential resources for human beings. Especially in India many families treat paddy as their daily food in the form of rice. Rice plays a key role in our day to day life; it is mostly consumed food in Asian countries. Like human beings, plants also get diseases, we humans can spell the symptoms but plants cannot so we need to observe carefully that if plant is facing from any disease. Due too many factors such as increasing in pollution, climatic changes, unseasonal rains different unknown diseases are affecting the rain plants. As the technology increasing, we need to identify and overcome the diseases in initial stage to reduce the loss of production. One of solution to identify problem is using Image recognition system, In these paper we propose a CNN algorithm for identifying three different types of rice diseases such as leaf smut, Bacterial Leaf Blight, Brown spot. This model got best accuracy compared to another algorithm and we also proposed an automated system to recognise paddy diseases by uploading the disease image.
\end{abstract}

Keywords - Paddy Diseases Images, Convolutional Neural Network, Python Programming Language.

\section{INTRODUCTION}

Agriculture describes the cultivation of crops along with an animal's husbandry, poultry and dairy farming. Among all over country the India is one of the biggest agricultural countries. Agriculture plays a crucial role in countries economy. Agriculture is the backbone for all over the world. Agriculture is processing the food by cultivating plants and growing them. Agriculture plays a vital role in the development of countries economy. Each and every human being daily consumes food to live. Paddy grows and follows some steps to become rice. Agriculture not only provides food and raw materials but also employment opportunities to very large proportion of population [1]. Two-Third of population is dependent on agriculture directly or indirectly. However the technology grows, the earth revolves us always stands behind the agriculture. Agriculture was the past, Agriculture is present and agriculture is our future. Many countries will grow

\author{
Jeevan Kumari Chevula \\ Department of Computer Science \\ SreeNidhi Institute of Science and Technology, HYD
}

paddy for not providing food but also to take major part in the country's economy. According to 2014 to 2019 survey figure 1 shows countries which are growing paddy in large amount. Based on survey China and Indian taken the top place in world in the rice production.

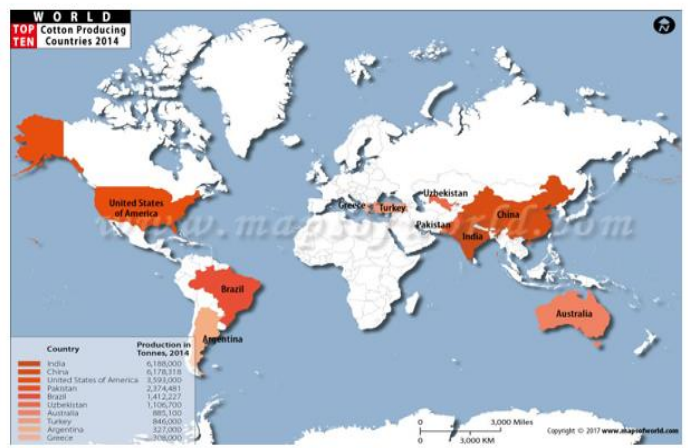

Fig 1: 2014-2019 Major Rice Producing Country

Rice diseases are rapidly increasing like human population, to improve the production we need to identify and overcome the diseases at the initial state.

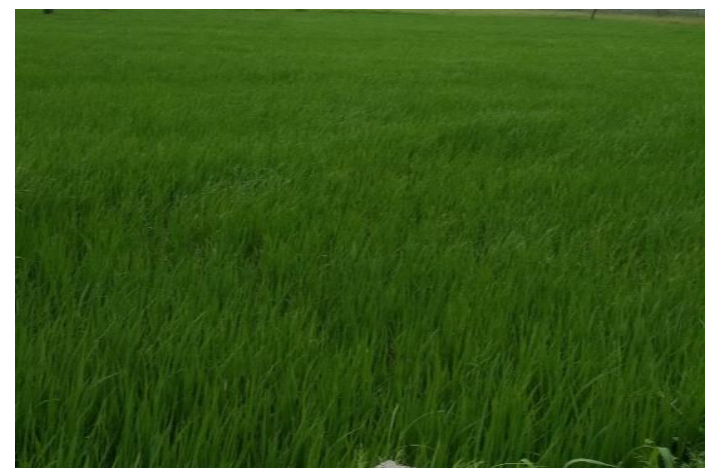

Fig 2: Healthy Paddy Crop

Mostly paddy crop required continuous flow of water, therefore there is more changes for growth and easily spread of diseases. Mostly diseases are caused by Bacteria, Fungus and Virus [2]. In this paper we come across only three different types of diseases are explained below:

\section{Bacterial Leaf Blight:}




\section{International Journal of Engineering Applied Sciences and Technology, 2020 \\ Vol. 5, Issue 3, ISSN No. 2455-2143, Pages 358-362 \\ Published Online July 2020 in IJEAST (http://www.ijeast.com)}

The disease was first observed in Japan (1884) and in India it was identified in the year 1959.In the year 1963, there is a severe damage by the BLB disease in Utter Pradesh and Bihar. There is about $50 \%$ yield loss occur by this disease. Godavari district in Andhra Pradesh is more affected by these diseases [3]. The bacterium (Xanthomonas Oryzae Bacteria) enters the plant through water pores along the edges of the leaf and through injuries in roots or leaves. The symptoms are Water soaked lesions on affected leaf, in the early morning we recognise Milky dew drops on leaves, Small yellowish beads can also be seen underneath the leaf, Leaf turns yellow to straw coloured and finally it will die. The favourable conditions for BLB is High use of Nitrogen, relative humidity above $70 \%$ and temperature at 25-34 degrees.
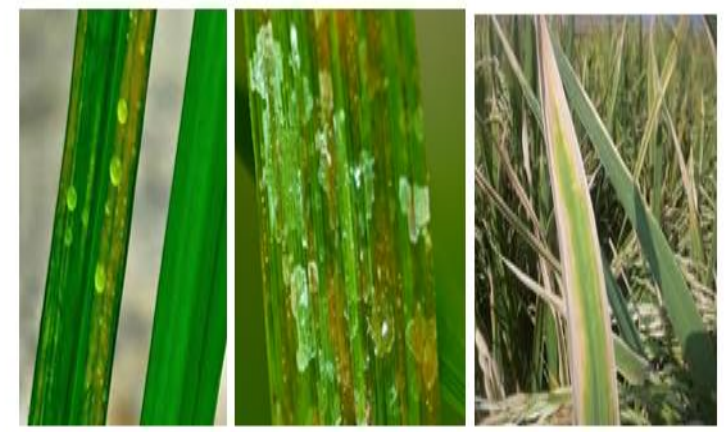

Fig 3: BLB Disease effected crop

Management: Grow resistant cultivars like MTU 9992, Swarna, Ajaya, IR 20, IR 42, IR 50, IR 54, TKM 6, Mashuri, IET 4141, IET 1444, IET 2508, Chinsura Boro, etc. Identify and burn the effected plant as early as possible and also reduce the use of nitrogenous fertilizers.

\section{Brown spot:}

It was first reported at MADRAS in 1919, caused by the Helminthosporium Oryzae fungus [4]. Typical spots on leaves are oval, they are usually thick dark brown spots relatively uniform and evenly distributed over the leaf surface. Most spots have light yellow halo having dark brown border looks like a sesame seeds. It is mainly spread by air-borne and seed borne. It occurs at all stages of plant, favourable conditions are 25-30 degrees temperature and humidity about $80 \%$.
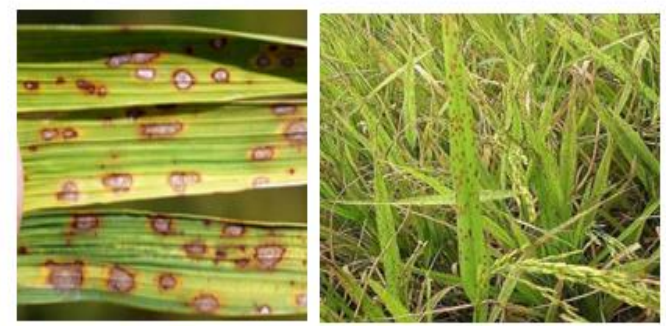

Fig 4: Brown Spot Disease effected crop
Management: Remove and burn affected plants in the soil and also spray once or twice with fungicides like $0.4 \%$ Bordeaux mixture or zineb@0.2\% or Copper oxy chloride@0.2\% or Mancozeb@0.25\%.

\section{Leaf smut:}

It was caused by Enthyloma Oryzae fungus, the main symptoms seen in leaf smut disease are 0.5-1.5 millimetres wide black spots. These spots are seen on both sides of leaves. The infected leaf has small size and full of black spots on .Heavily infected leaves turn yellow and the leaf tips die.
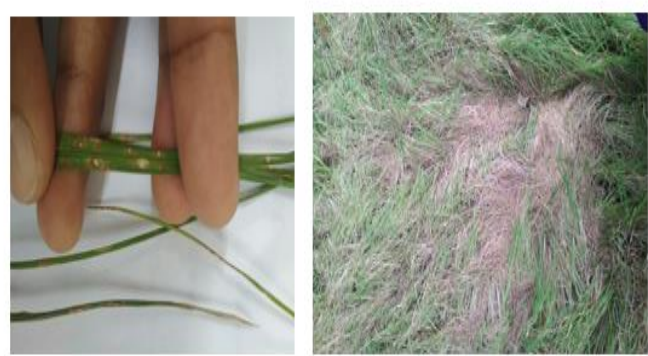

Fig 5: Leaf Smut Disease effected crop

This research paper is organised as follows: Segment -2 presents the Literature Survey, Segment-3 have Proposed Work, Segment-4 contains Implementation and Results, Segment-5 contains Conclusion, and Segment-6 contains Acknowledgment following References.

\section{RELATED WORK}

\section{Prediction of Rice Diseases Using Convolutional Neural Network (in Rstudio)}

Prediction of Rice diseases using Convolutional Neural Network [5], in this paper predicting the disease of rice using $r$ language taken some dataset with less volume of data and predicted the rice disease using Convolutional neural network algorithm. The accuracy of an algorithm used in an $r$ language comes up with the result of $74 \%$. Rice is mostly consumed largest food product across the world. While growing the rice or an paddy plants occurs some different types of diseases which was caused by the fungus, bacteria, mosquitos etc. For predicting the disease which caused to the rice plants used CNN algorithm method by capturing the rice leaves images. It says about the disease of a rice plants and how much percentage it occurs. CNN algorithm plays important role in detecting diseases of a rice plants with that images. CNN algorithm reduces the time by reducing the parameters. This algorithm follows some layers Convolution, ReLu Layer, Pooling Layer, and Fully Connected which are implemented in R Programming Language.

Rice Blast Disease Recognition Using a Deep Convolutional Neural Network 


\section{International Journal of Engineering Applied Sciences and Technology, 2020 \\ Vol. 5, Issue 3, ISSN No. 2455-2143, Pages 358-362 \\ Published Online July 2020 in IJEAST (http://www.ijeast.com)}

Rice Blast Disease Recognition Using a Deep Convolutional Neural Network [6], Wan-jie Liang, Hong Zahang, Gu-feng Zahang, Hong-xin Cao implemented a method identification of rice blast disease only, they got have accuracy of about $80 \%$ in the training dataset. The dataset consists of 2906 positive samples and 2902 negative samples is collected for training and testing the $\mathrm{CNN}$ model. In this paper the rice images with rice blast diseases are collected from the Institute of Plant Protection, Jiangsu Academy of Agricultural Sciences, and Nanjing, China. CNN model is a multi-layer neural network which was a supervised learning architecture it is made up two parts with the features of extractor and a trainable and a trainable classifier. CNN has two important properties one is local receptive field and other one is shared weights. To identify the relationship between pixels and raw images the convolutional filtering used. The accuracy of this method obtained is $80 \%$.

\section{PROPOSED WORK}

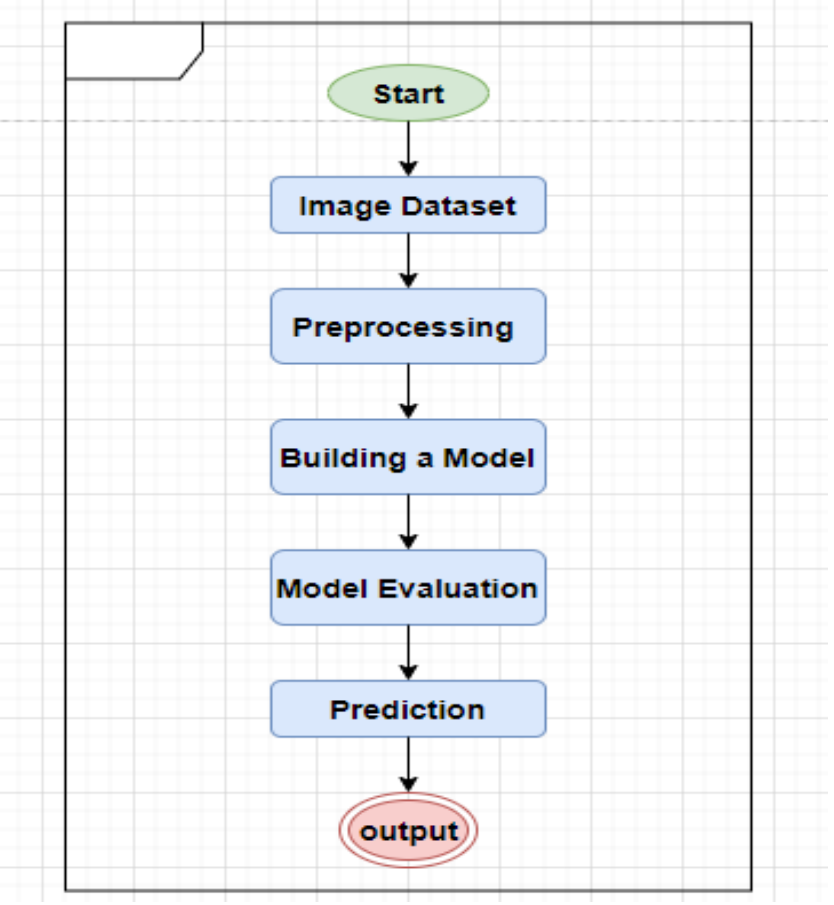

Fig 6: shows the architectural model of the proposed work

We collected the Image dataset of three diseases namely BLB, Brown spot, Leaf smut from UCI Machine Learning Repository [7]. To increase the prediction rate, we flipped the images [8] and stored in same dataset total as 180 samples.

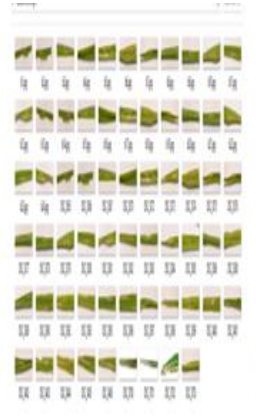

Bacterial Leaf Blight

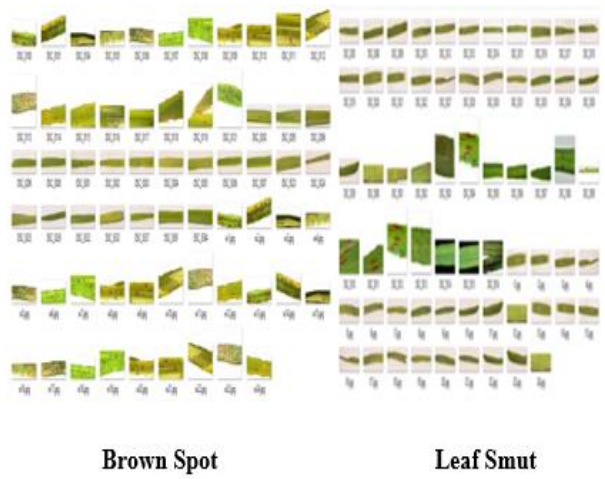

Fig 7: Infected Paddy leaf images of Dataset Sample

Then we perform pre-processing steps such as converting the images into Grayscale [9], resizing and reshaping the images. After that we build a model using Convolutional Neural Network.

Convolutional Neural Network:

Actually computer understand the images based on the pixel values, for colour image there are three channel RGB and for Black and white have two channels.

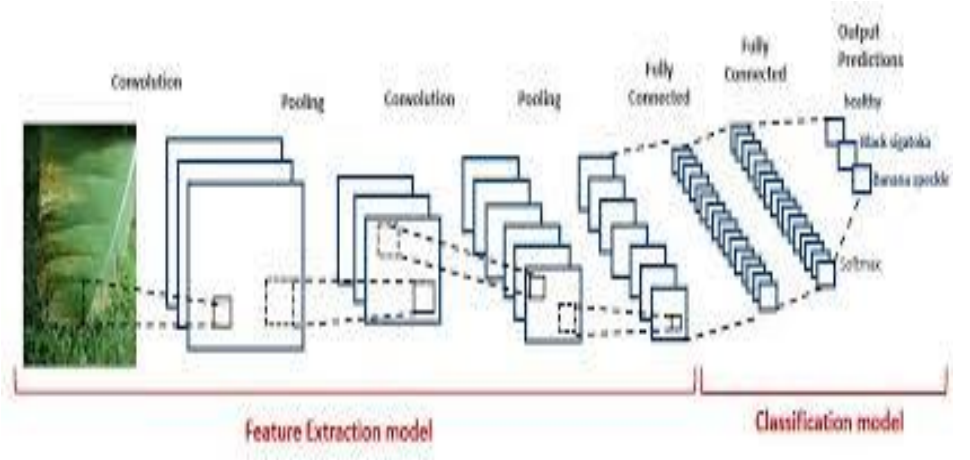

Fig 8: Working of $\mathrm{CNN}$

CNN or ConvNet is a type of feed-forward artificial neural network in which the connected pattern is inspired by the animal visual cortex [10].

Working of CNN: It have following layers:-

1. Convolution Layer

2. ReLU Layer

3. Pooling Layer

4. Fully Connected Layer

CNN compares the images piece by piece, the piece that it looks for are called features or filters [11].

In Convolutional layer it multiple the filters with entire image pixels and in ReLU it remove every negative values from the filtered images and replaces it with zeros. 


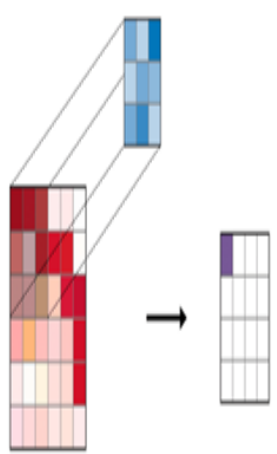

Convolutional layer

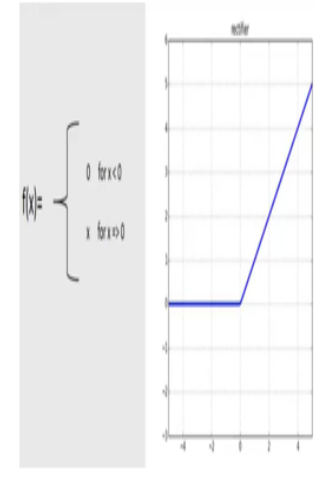

ReLU layer
Pooling layer, we reduce size of image by replace the window with the highest values. The actual classification happens in Fully Connected Layer, here we take our filtered and shrinked images and put them into a single list.

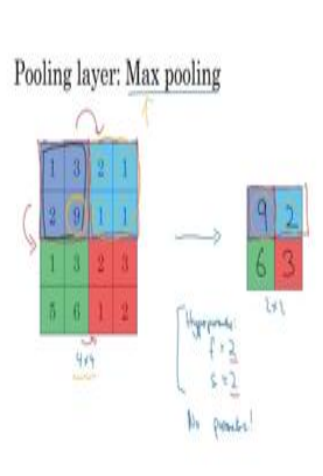

Pooling Layer

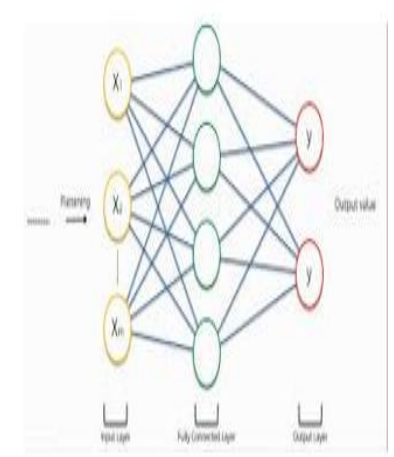

Fully comnected Layer

\section{IMPLEMENTATION}

In these project main theme is to identify paddy disease type based on the input given image

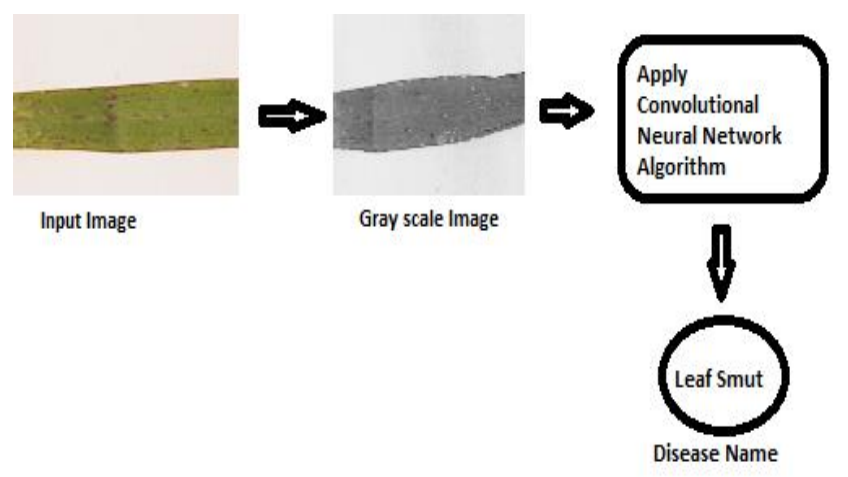

Fig 9: View of project
Gray Scale conversion reduce the image pixels and make convenient to run algorithm, these project was implemented in python framework. We pass images through Convolutional layers, in these we gone through Max Pooling layer and Activation function.

\begin{tabular}{|c|c|c|}
\hline Layer (type) & Output Shape & Param \# \\
\hline conv2d_15 (Conv2D) & (None, 48, 48, 32) & 320 \\
\hline activation_30 (Activation) & (None, 48, 48, 32) & 0 \\
\hline $\max$ pooling2d 15 (MaxPooling & (None, 24, 24, 32) & 0 \\
\hline conv2d_16 (Conv2D) & (None, 22, 22, 64) & 18496 \\
\hline activation_31 (Activation) & (None, 22, 22, 64) & 0 \\
\hline max_pooling2d_16 (MaxPooling & (None, 11, 11, 64) & 0 \\
\hline $\begin{array}{ll}\text { conv2d } 17 \text { (Conv2D) }\end{array}$ & (None, 9, 9, 64) & 36928 \\
\hline activation 32 (Activation) & (None, 9, 9, 64) & 0 \\
\hline max_pooling2d_17 (MaxPooling & (None, 4, 4, 64) & 0 \\
\hline dropout_5 (Dropout) & (None, 4, 4, 64) & 0 \\
\hline flatten 5 (Flatten) & (None, 1024) & 0 \\
\hline dense 15 (Dense) & (None, 128) & 131200 \\
\hline activation_33 (Activation) & (None, 128) & 0 \\
\hline dense_16 (Dense) & (None, 128) & 16512 \\
\hline activation_34 (Activation) & (None, 128) & 0 \\
\hline dense 17 (Dense) & (None, 13) & 1677 \\
\hline activation_35 (Activation) & (None, 13) & 0 \\
\hline
\end{tabular}

Fig 10: Model Summary

In a CNN, each layer has two kinds of parameters such as Weights and biases. The total number of params are just the sum of all parameters.

There will no params in Max Pooling and Activation layers, in the given above model shows there are 205,133 trainable params. Flatten layer transforms two dimensional matrix into a simple vector. Dense layer is simply used to connect the input node to the output node densely and finally Dropout layer is used to prevent overfitting.

\section{RESULT ANALYSIS}

In these project we got an accuracy of $90 \%$, to improve accuracy we need to train more images.

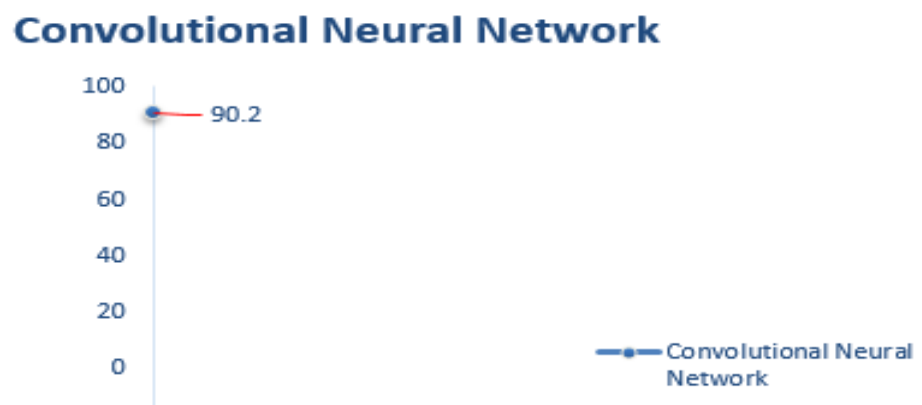

Fig 11: Accuracy 


\section{REFERENCE}

Out[9]: 〈matplotlib.legend. Legend at ex719fe49f98〉

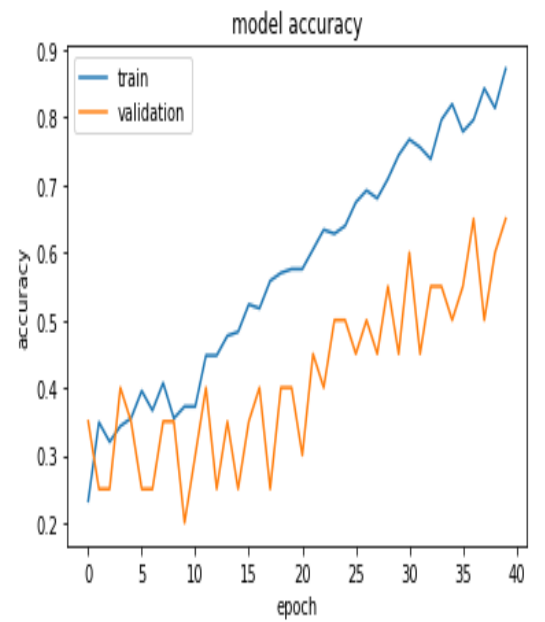

Fig 12: Model Accuracy

Here blue colour line shows the training set accuracy and orange line shows the validation set.

To test the model, we passed an infected leaf image. Given below shows predicted result using Convolutional Neural Network.
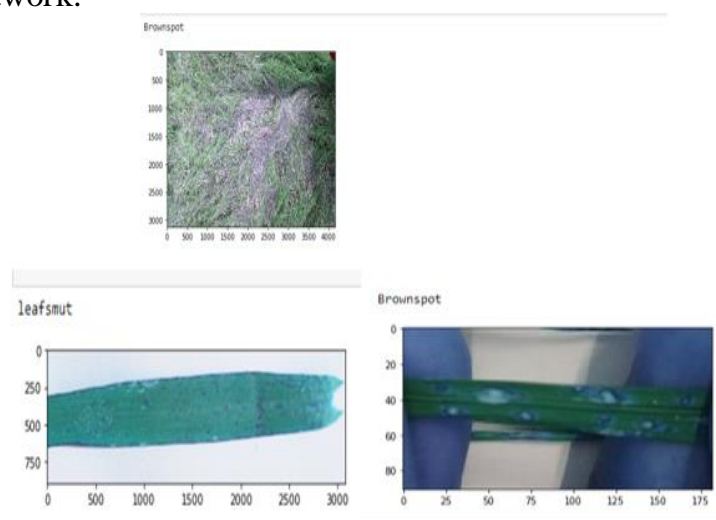

Fig 13: Output

In the above results, it is correctly predicted the disease names of Leaf Smut and Brown spot.

\section{CONCLUSION}

These system helps to identify disease by uploading infected images, there is lot of research require in the dataset because it plays a crucial role for best results. Compared to all the algorithms CNN got high accuracy. There is urgent need to improve the research in agriculture for future generations. Identifying the diseases at initial stage will helps to overcome crop damage and increase production.
[1] "Classificatiion rules for Indian Rice Diseases",IJCSI International Journal Of Computer Science Issues, VOL.8,ISNAL SUE, January 2011.

[2] "Decision Tree-Based Machine Learning Algorithmsto classify rice plant diseases", Internal Journal Of Innovative Technology and Exploring Engineering(IJITEE)ISSSN: 2278-3075,Volume-9 Issue1, November 2019.

[3] Francisco Elazegui, and Zahirul Islam, "Diagnosis of common diseases of rice," International Rice Research Institute, (C) 2003.

[4] https://www.lsuagcenter.com/ /media/system/4/6/2/f/462f d54617eaff51c0fdeb50d5feccdd/pub3108brownspothighr es.pdf.

[5] "Prediction of Rice Diseases Using Convolutional Neural Network (in Rstudio)", International Journal of Innovative Science and Research Technology, Volume 4, Issue 12, December - 2019.

[6] "Rice Blast Disease Recognition Using a Deep ConvolutionalNeuralNetwork",www.nature.com/scientifc reports, published on 27 Feb 2019.

[7] https://archive.ics.uci.edu/ml/datasets/Rice+Leaf+Diseass.

[8] Detection and Classification of Rice plant disease", Research Gate, Journal: Intelligent Decision Technologies, 2017 volume 11 no 3, pp 357-373.

[9] "Leaf disease detection using image processing", Journal of Chemical and Pharmaceutical Sciences, published on 23 November 2017.

[10] "Identification of Rice Diseases using Deep Convolutional Neural Network", Elsevier- 2017.

[11] "SMART PADDY CROP DISEASE IDENTIFICATION AND MANAGEMENT USING DEEP CONVOLUTION NEURAL NETWORK AND SVM CLASSIFIER", International Journal of Pure and Applied Mathematics Volume 118 No. 15 2018, 255264.

\section{AUTHORS PROFILE}

Sony Annem has been pursuing M.Tech in Computer Science Engineering, Sreenidhi Institute of Science and Technology. She has received her B.Tech (Computer Science Engineering) degree from Vijaya Engineering College. Her areas of interest are Data Mining and Machine Learning.

JeevankumariChevula has been pursuing M.Tech in Computer Science Engineering, Sreenidhi Institute of Science and Technology. She has received her B.Tech(Information Technology) degree from Megha Institute of Engineering and Technology for women. Her areas of interest are Machine Learning, Artifcial Intelligences, Data Mining,Cryptography. 